\title{
Classification of composite semantic relations by a distributional- relational model
}

\author{
Siamak Barzegar ${ }^{\mathrm{a}, *}$, Brian Davis ${ }^{\mathrm{b}}$, Siegfried Handschuh ${ }^{\mathrm{c}}$, Andre Freitas ${ }^{\mathrm{d}}$ \\ a Insight Centre for Data Analytics, National University of Ireland, Galway, Ireland \\ ${ }^{\mathrm{b}}$ Department of Computer Science, Maynooth University, Ireland \\ ${ }^{\mathrm{c}}$ Department of Mathematics and Computer Science, University of Passau, Germany \\ ${ }^{\mathrm{d}}$ School of Computer Science, The University of Manchester, UK
}

\section{A R T I C L E I N F O}

\section{Keywords:}

Semantic relation

Distributional semantic

Deep learning

Classification

\begin{abstract}
A B S T R A C T
Different semantic interpretation tasks such as text entailment and question answering require the classification of semantic relations between terms or entities within text. However, in most cases, it is not possible to assign a direct semantic relation between entities/terms. This paper proposes an approach for composite semantic relation classification using one or more relations between entities/term mentions, extending the traditional semantic relation classification task. The proposed model is different from existing approaches which typically use machine learning models built over lexical and distributional word vector features in that is uses a combination of a large commonsense knowledge base of binary relations, a distributional navigational algorithm and sequence classification to provide a solution for the composite semantic relation classification problem. The proposed approach outperformed existing baselines with regard to F1-score, Accuracy, Precision and Recall.
\end{abstract}

\section{Introduction}

Capturing the semantic relationship between two concepts is a fundamental operation for many semantic interpretation tasks. This is a task which humans perform rapidly and reliably by using their linguistic and commonsense knowledge about entities and relations. Natural Language Processing $(N L P)$ systems which aspire to reach the goal of producing meaningful representations of text must be equipped to identify and learn semantic relations in the documents they process. The automatic recognition of semantic relations has many applications such as information extraction, document summarization, machine translation, or the construction of thesauri and semantic networks. It can also facilitate auxiliary tasks such as word sense disambiguation, language modelling, paraphrasing, and recognizing textual entailment [25].

However, it is not always possible to establish a direct semantic relation given two entity mentions in text. In the SemEval 2010 Task 8 test collection [25] for example, $17.39 \%$ of the semantic relations mapped within sentences were assigned with the label OTHER, meaning that they could not be mapped to the set of 9 direct semantic relations. ${ }^{1}$ In many cases, the semantic relations between two entities can only be expressed by a composition of two or more operations. For example, there is no direct relation

\footnotetext{
* Corresponding author.

E-mail addresses: siamak.barzegar@insight-centre.org (S. Barzegar), brian.davis@mu.ie (B. Davis), Siegfried.Handschuh@uni-passau.de (S. Handschuh), andre.freitas@manchester.ac.uk (A. Freitas).

${ }^{1}$ Cause-Effect, Instrument-Agency, Product-Producer, Content-Container, Entity-Origin, Entity-Destination, Component-Whole, MemberCollection, Communication-Topic.
} 
between Engineer and college within current knowledge graph or semantic networks such as DBpedia or ConceptNet.

This work aims at improving the description and the formalization of the semantic relation classification task, in which the relations between entities can be expressed using the composition of one or more relations.

This paper is organized as follows: Section 2 describes the semantic relation classification problem and the related work (Section 3). Section 4.3 describes the existing baseline models, while Section 4 describes the experimental setup and analyses the results, providing a comparative analysis between the proposed model and the baselines. Finally, Section 5 offers a conclusion.

\section{Composite semantic relation classification}

\subsection{Semantic relation classification}

Semantic relation classification is the task of classifying the underlying abstract semantic relations between target entities (terms) present in texts [36]. The goal of relation classification is defined as follows: given a sentence $S$ with pairs of annotated target nominals $e_{1}$ and $e_{2}$, the relation classification system aims to classify the relations between $e_{1}$ and $e_{2}$ in given texts within the predefined relation set [25]. For instance, the relation between the nominal burst and pressure in the following example sentence is interpreted as Cause-Effect $\left(e_{2}, e_{1}\right)$.

The $<e_{1}>$ burst $</ e_{1}>$ has been caused by water hammer $<e_{2}>$ pressure $</ e_{2}>$.

SemEval 2010 Task 8 [25] focuses on Multi-Way classification of semantic relations between pairs of nominals. For instance, student and association are in a Member-Collection relation in "The student association is the voice of the undergraduate student population of the State University of New York at Buffalo".

They selected nine general relations plus "OTHER" is as follows:

- Cause-Effect. An event or object leads to an effect. Example: $<e_{1}>$ Smoking $</ e_{1}>$ causes $<e_{2}>$ cancer $</ e_{2}>$.

- Instrument-Agency. An agent uses an instrument. Example: $\left\langle e_{1}>\right.$ Laser $\left.<e /{ }_{1}\right\rangle<e_{2}>$ printer $</ e_{2}>$

- Product-Producer. A producer causes a product to exist. Example: The $\left\langle e_{1}>\right.$ farmer $</ e_{1}>$ grows $<e_{2}>$ apples $</ e_{2}>$

- Content-Container. An object is physically stored in a delineated area of space, the container. Example: $\left\langle e_{1}\right\rangle$ Earth $\left.</ e_{1}\right\rangle$ is located in the $<e_{2}>$ MilkyWay $</ e_{2}>$.

- Entity-Origin. An entity is coming or is derived from an origin (e.g., position or material). Example: $<e_{1}>$ Letters $</ e_{1}>$ from $<e_{2}>$ foreigncountries $</ e_{2}>$.

- Entity-Destination. An entity is moving towards a destination. Example: The $\left\langle e_{1}>\right.$ boy $</ e_{1}>$ went to $<e_{2}>$ bed $</ e_{2}>$.

- Component-Whole. An object is a component of a larger whole. Example: $\mathrm{My}<e_{1}>$ apartment $</ e_{1}>$ has a large $<$ $e_{2}>$ kitchen $</ e_{2}>$.

- Member-Collection. A member forms a nonfunctional part of a collection. Example: There are many $<e_{1}>$ trees $</ e_{1}>$ in the $<e_{2}>$ forest $/<e_{2}>$.

- Communication-Topic. An act of communication, whether written or spoken, is about a topic. Example: The $<e_{1}>$ lecture $</ e_{1}>$ was about $<e_{2}>$ semantics $</ e_{2}>$.

The final dataset contains a set of 10, 717 instances, where 8, 000 instances are defined as the training set. Table 1 shows the distribution of categories for the dataset. The second column (Frequency) shows the absolute and relative frequencies of each relation.

Table 1

Annotation Statistics of relation types with absolute and relative frequency in the dataset.

\begin{tabular}{ll}
\hline Relation & Frequency \\
\hline Cause-Effect & $1331(12.4 \%)$ \\
Component-Whole & $1253(11.7 \%)$ \\
Entity-Destination & $1137(10.6 \%)$ \\
Entity-Origin & $974(9.1 \%)$ \\
Product-Producer & $948(8.8 \%)$ \\
Member-Collection & $923(8.6 \%)$ \\
Message-Topic & $895(8.4 \%)$ \\
Content-Container & $732(6.8 \%)$ \\
Instrument-Agency & $660(6.2 \%)$ \\
Other & $1864(17.4 \%)$ \\
\hline Total & $10717(100 \%)$ \\
\hline
\end{tabular}




\subsection{Existing approaches for single semantic relation classification}

Different approaches have been explored for relation classification, including unsupervised and supervised relation discovery and classification. Existing literature has proposed various features to identify the relations between entities using different methods, which are described in the following paragraphs.

In the unsupervised methods, contextual features are used. The distributional hypothesis [23] indicates that words that have similar meanings, probably occur in the same context. Accordingly, it is assumed that the pairs of words that occur in similar contexts tend to have similar relations. Hasegawa et al. [24] used the contexts of nominal words using a hierarchical clustering method which represents the relationship between the words by using the most frequent words in the contexts. Chen et al. [9] suggested an unsupervised algorithm for addressing this problem based on model-order selection and discriminative label identification.

In the supervised methods, approaches can be grouped into two types: feature-based and kernel-based (see Ref. [50] for more details). The performance of these models strongly depends on the quality of the designed features. Recently, Neural network-based approaches have achieved significant improvement over traditional methods based on human-designed features [36]. Existing neural networks for relation classification are usually based on shallow architectures (e.g., one-layer convolutional neural networks or recurrent networks). In exploring the potential representation space at different abstraction levels, they may fail to perform [47]. The performance of supervised approaches strongly depends on the quality of the designed features [50]. Complementarily, some models are exploring automatic feature learning strategies. Xu et al. [48] introduce gated recurrent networks, in particular, Long Short-Term Memories (LSTMs) applied to relation classification. Zeng et al. [50] use Convolutional Neural Networks (CNNs) for the same task. Additionally, Santos et al. [38] replace the common Softmax loss function with a ranking loss in their CNN model. Xu et al. [46] design a negative sampling method based on CNNs. From the viewpoint of model ensembles, Liu et al. [30] combine CNNs and recursive networks along the Shortest Dependency Path (SDP), while Nguyen et al. [34] incorporate CNNs with Recurrent Neural Networks (RNNs).

Additionally, much effort has been invested in relational learning methods that can scale to large knowledge bases. The best performing neural-embedding models are Socher et al. (NTN) [41] and Bordes et al. models (TransE and TATEC) [8,21].

\section{From single to composite relation classification}

\subsection{Introduction}

The goal of this work is to propose an approach for semantic relation classification using one or more relations between entities/ term mentions. In below example, the relationship between Child and Cradle cannot be directly expressed by one of the nine abstract semantic relations from the set described in Ref. [25].

The $<e_{1}>$ child $</ e_{1}>$ was carefully wrapped and bound into the $<e_{2}>$ cradle $</ e_{2}>$ by means of a cord.

Assume $R_{1}$ be a relation from $X$ to $Y$, and $R_{2}$ be a relation from $Y$ to $Z$. Then a relation written as $R_{1} \bigcirc R_{2}$ is called a composite relation of $R_{1}$ and $R_{2}$ where

$$
\left.R_{1} \circ R_{2}=(x, y) \mid x \in X \wedge z \in Z \wedge(\exists y)\left(y \in Y \wedge(x, y) \in R_{1} \wedge(y, z) \in R_{2}\right)\right)
$$

We can also write the composition as

$$
R_{1} \circ R_{2}=(x, y) \mid x \in X \wedge z \in Z \wedge(\exists y)\left(y \in Y \wedge x R_{1} y \wedge y R_{2} z\right)
$$

Note: Relational composition can be realized as matrix multiplication. For example, let $M_{R_{1}}$ and $M_{R_{2}}$ represent the binary relations $R_{1}$ and $R_{2}$, respectively. Then $R_{1} \bigcirc R_{2}$ can be computed via $M_{R_{1}} M_{R_{2}}$.

Based on the definition of Composition of relation has been explained and looking into a commonsense KB (in this case, ConceptNet V 5.4) we can see the following set of composite relations between these elements:

$$
<e_{1}>\text { child }</ e_{1}>\text { CreatedByoCausesoAtLocation }<e_{2}>\text { cradle }</ e_{2}>
$$

With the increase in the number of edges which can be included in the set of semantic relation compositions (the size of the semantic relationship path), there is a dramatic increase in the number of paths which connect the two entities. For example, for the words child and cradle there are 15 paths of size 2 , " 1,079 " paths of size 3 and " 95,380 " paths of size 4 . Additionally, as the path size grows many non-relevant relationships (less meaningful or redundant relations) will be included.

The challenge in composite semantic relation classification is to provide a classification method that provides the most meaningful (see more details on Section 3.3) set of relations for the context at hand. This task can be challenging because, as previously mentioned, a simple KB lookup based approach would provide all semantic associations at hand. To achieve this goal we propose an approach which combines sequence-based machine learning models, distributional semantic models and commonsense relational knowledge bases to provide an accurate method for composite semantic relation classification. The proposed model (Fig. 1) relies on the combination of the following approaches:

i Using existing structured commonsense KBs to define an initial set of semantic relation compositions.

ii Using a pre-filtering method based on the Distributional Navigational Algorithm (DNA) as proposed by Refs. [20,39].

iii Using a sequence-based Neural Network based model to quantify the sequence probabilities of the semantic relation compositions.

We call this model Neural Concept-Relation Model; an analogy to a Language Model. 

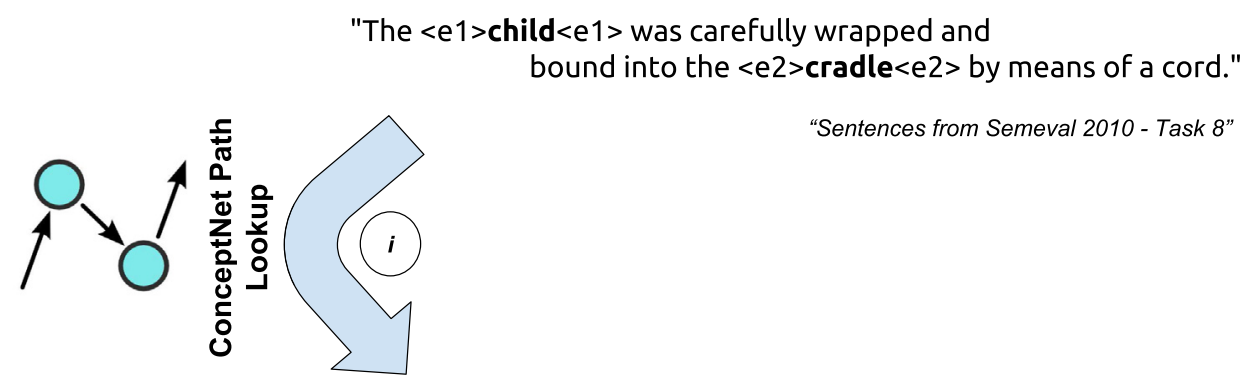

"Sentences from Semeval 2010 - Task 8"

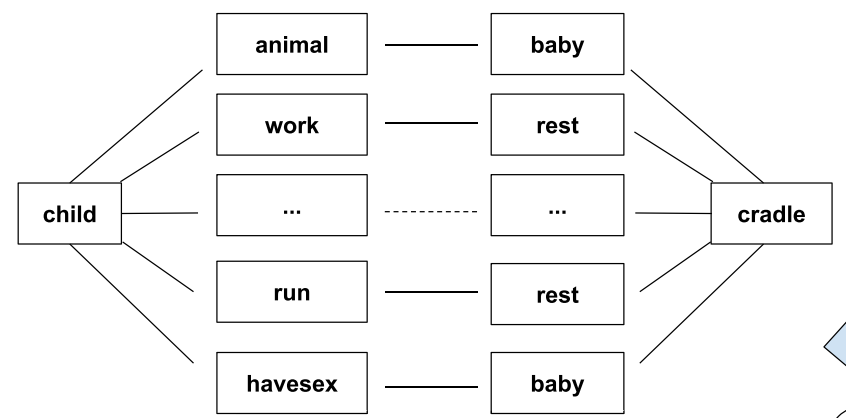

1094 paths of size 1,2 and 3
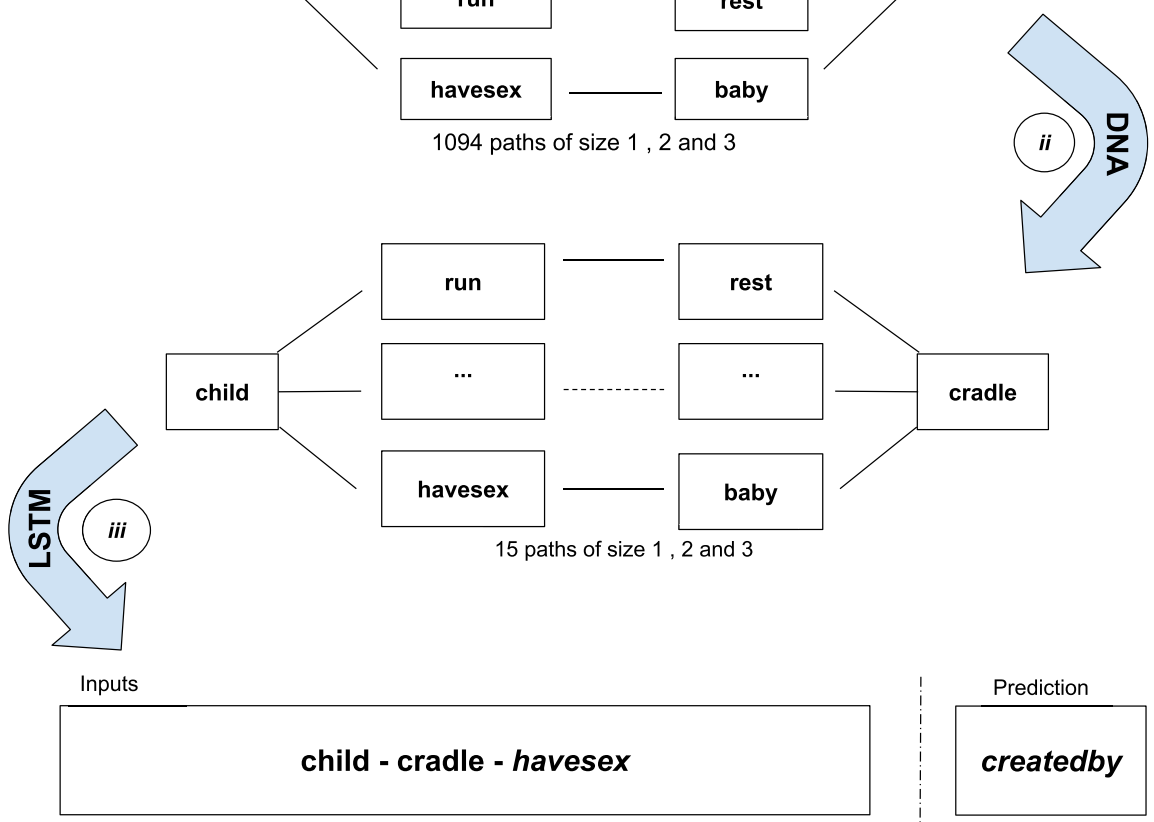

child - cradle - baby - havesex - creadby
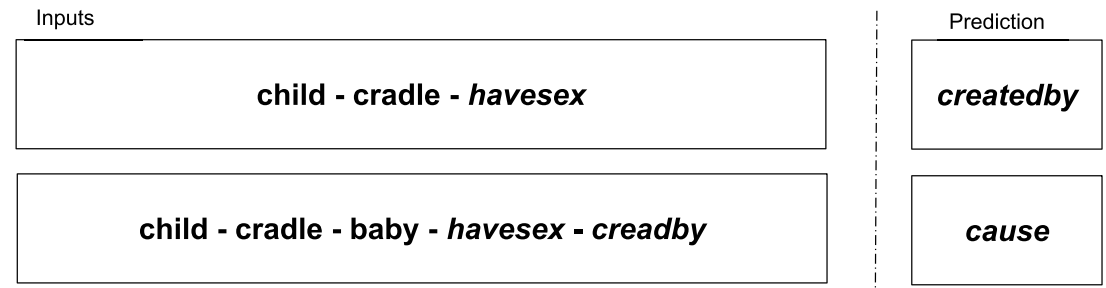

child - cradle - baby - cause - havesex - createdby

cause

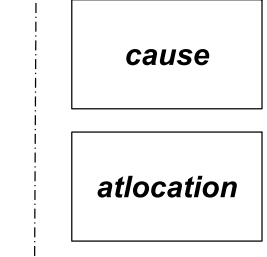

Fig. 1. Depiction of the proposed model relies on the combination of our three approaches.

\subsection{Commonsense KB lookup}

The first step consists in the use of a large commonsense knowledge base for providing a reference for a sequence of semantic relations. ConceptNet [44] is a semantic network built from existing linguistic resources and crowd-sourced. It is built from nodes representing words or short phrases as observed in natural language and labelled abstract relationships between them. There are a few alternative for large commonsense KBs, such as WordNet, Microsoft Concept Graph and DBpedia. In the list below, ConceptNet is contrasted to other KBs: 
- WordNet: ConceptNet has more relation types than WordNet. Additionally, ConceptNet's vocabulary ${ }^{2}$ is much larger and contains more links between the Concepts. ConceptNet does not assume that words fall into synsets. Furthermore, synonymy in ConceptNet is a relation like any other. ConceptNet reuses some relations from WordNet. Moreover, their importance is weighted higher, given that the knowledge in WordNet is handcrafted, accurate and of high quality.

- Microsoft Concept Graph: Microsoft Concept Graph is a taxonomy of English nouns ${ }^{3}$ containing IsA relations

- DBpedia: DBpedia is focused on named entities and basic factoid-style attributes. In contrast,ConceptNet focuses on noun/verblevel entities and their abstract relations.

ConceptNet is used as a large commonsense knowledge base for the proposed model. The intuition is that any type of relation classification task would need to be based on large-scale commonsense knowledge either in a distributional or structured/relational form. ConceptNet ${ }^{4}$ has been built from several sources, such as:

- Information extracted from parsing Wiktionary [51]

- Open Multilingual WordNet [7]

- Open Mind Common Sense [40]

- A subset of DBpedia [1]

ConceptNet has a long-trail distribution of relations. However, the more frequent relations expressed at ConceptNet are ${ }^{5}$ :

- Symmetric relations: Antonym, DistinctFrom, EtymologicallyRelatedTo, LocatedNear, RelatedTo, SimilarTo, and Synonym.

- Asymmetric relations: AtLocation, CapableOf, Causes, CausesDesire, CreatedBy, DefinedAs, DerivedFrom, Desires, Entails, ExternalURL, FormOf, HasA, HasContext, HasFirstSubevent, HasLastSubevent, HasPrerequisite, HasProperty, InstanceOf, IsA, MadeOf, MannerOf, MotivatedByGoal, ObstructedBy, PartOf, ReceivesAction, SenseOf, SymbolOf, and UsedFor.

For our target example, 1, 094 paths were extracted from ConceptNetfor two given entities (e.g. child and cradle) such that they contained no corresponding semantic relation from the SemEval 2010 Task 8 test collection (Fig. 1(i)). Examples of paths are ${ }^{6}$ :

- child/CanBe/baby/AtLocation/cradle

- child/IsA/animal/HasA/baby/AtLocation/cradle

- child/HasProperty/work/CausesDesire/rest/Synonym/cradle

- child/InstanceOf/person/Desires/baby/AtLocation/cradle

- child/DesireOf/run/CausesDesire/rest/Synonym/cradle

- child/CreatedBy/havesex/Causes/baby/AtLocation/cradle

Although ConceptNet can provide a large commonsense Knowledge Base, as we transcend immediate single relations, paths start to conceptually drift away from the source and target concepts. In order to filter these relations into a set of semantically relevant paths, we apply the Distributional Navigational Algorithm (DNA), described in the next section.

\subsection{Distributional navigational algorithm (DNA)}

The Distributional Navigational Algorithm (DNA) consists of an approach which uses distributional semantic models as a relevance-based heuristic for selecting relevant facts attached to a contextual query over a structured KB. DNA provides an abductive reasoning style mechanism which operates over Distributional-Relation Models [14,16-19], i.e. models which enrich structured logical (triple-style) KBs with word-embedding style information.

The DNA approach focuses on addressing the following problems: (i) providing a semantic selection mechanism for facts which are relevant and meaningful in a particular reasoning \& querying context and (ii) allowing coping with information incompleteness in large KBs. The DNA model starts from the source entity and navigates through the KB, computing the distributional semantic relatedness between the set of lexical elements associated with neighbouring nodes in the graph and the target entity. The semantic relatedness function is defined as:

$$
\operatorname{sr}\left(\overrightarrow{p_{1}}, \overrightarrow{p_{2}}\right)=\cos (\theta)=\overrightarrow{p_{1}} \cdot \overrightarrow{p_{2}}
$$

where $s r: V S^{\text {dist }} \times V S^{\text {dist }} \rightarrow[0,1]$.

An important point to emphasize is the fact that the distributional semantic relatedness function is defined over an external/ independent corpus (in contrast to many existing approaches which define the embeddings based on the KB). The DNA method is not

\footnotetext{
228 million statements.

35.4 million concepts.

${ }^{4}$ Version 5.5.

5 https://github.com/commonsense/conceptnet5/wiki/Relations.

${ }^{6}$ Paths in bold are considered semantically relevant.
} 
coupled to a specific distributional/word embedding model and can use different types of models.

A threshold $\eta \in[0,1]$ can be used to establish the desired semantic relatedness between two vectors: $\operatorname{sr}\left(\overrightarrow{p_{1}}, \overrightarrow{p_{2}}\right)>\eta$. The information provided by the semantic relatedness function $s r$ is used to identify elements in the KB with a similar meaning from the reference corpus perspective. The threshold is calculated following the semantic differential approach proposed in Ref. [20]. Multiword phrases are handled by calculating the centroid between the concept vectors defined by each word in the Distributional Navigation Algorithm (DNA) (Algorithm 1) [20,39].

In summary, given two semantically related terms source and target wrt a threshold $\eta$, the algorithm finds all paths from source to target, with length $l$, formed by concepts semantically related to target wrt $\eta$.

The source term is the first element in all paths. From the set of paths to be explored (ExplorePaths), the DNA selects a path and expands it with all neighbours of the last term in the selected path that are semantically related wrt the threshold $\eta$ and but do not appear in that path. The stop condition is sr(target, target) $=1$ or when the maximum path length is reached.

The paths $p=\left\langle t_{0}, t_{1}, \ldots, t_{l}\right\rangle$ (where $t_{0}=$ source and $t_{l}=$ target) found by DNA are ranked (line 14 of code) according to the following formula:

$$
\operatorname{rank}(p)=\sum_{i=0}^{l} \operatorname{sr}\left(\overrightarrow{t_{i}}, \overrightarrow{\text { target }}\right)
$$

Algorithm 1 can be modified to use a heuristic that allows to expand only the paths for which the semantic relatedness between all the nodes in the path and the target term increases along the path. The differential in the semantic relatedness for two consecutive iterations is defined as $\Delta \operatorname{target}\left(t_{1}, t_{2}\right)=\operatorname{sr}\left(\overrightarrow{t_{2}}, \overrightarrow{\text { target }}\right)-\operatorname{sr}\left(\overrightarrow{t_{1}}, \overrightarrow{\text { target }}\right)$, for terms $t_{1}, t_{2}$ and target. This heuristic is implemented by including an extra test in the line 7 condition, i.e., $\Delta \operatorname{target}\left(t_{k}, n\right)>0$.

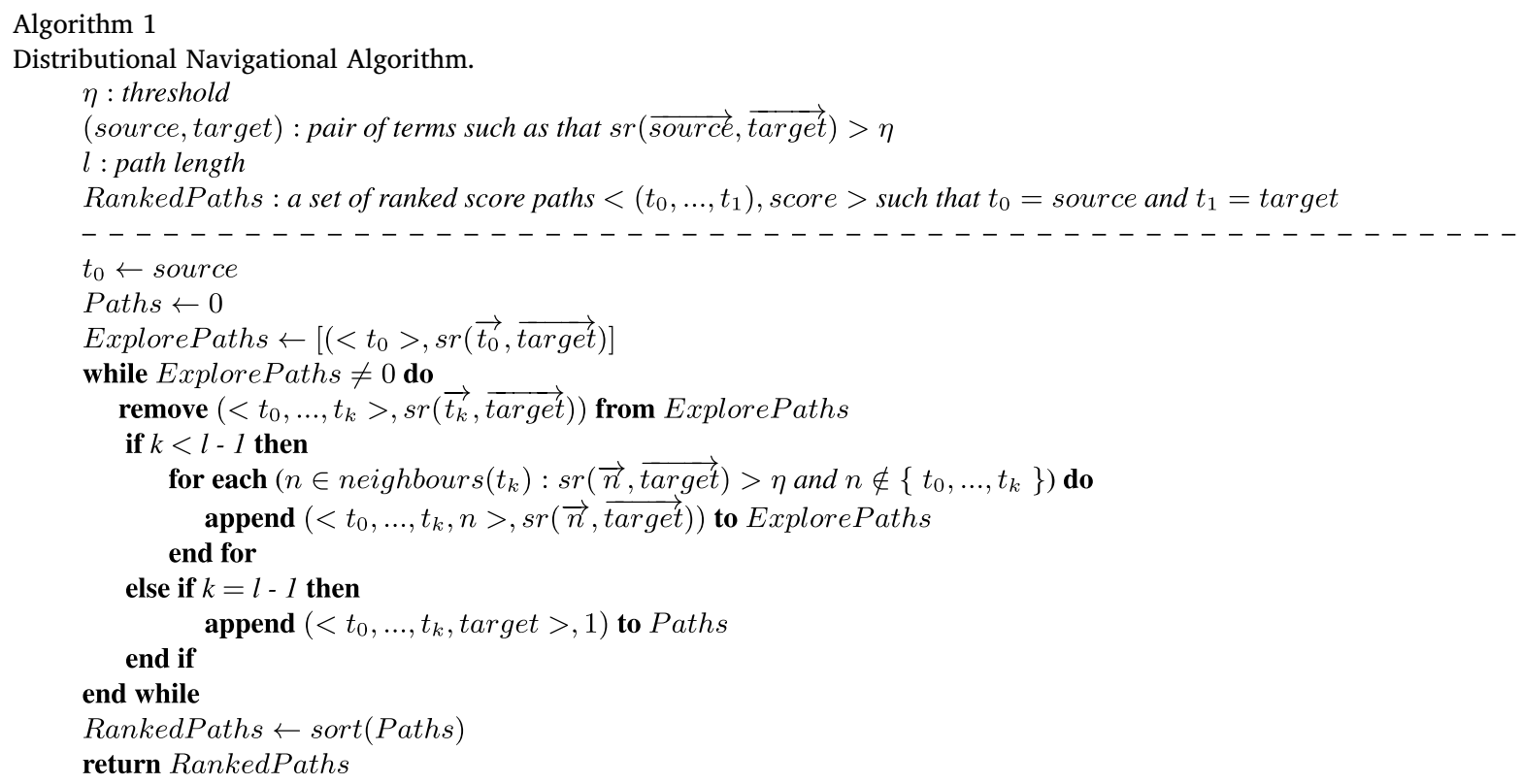

In Ref. [20], DSMs are used as a complementary semantic layer to the relational model, which supports coping with semantic approximation and incompleteness. For large-scale and open domain commonsense reasoning scenarios, model completeness, and full materialization cannot be assumed. A commonsense KB would contain vast amounts of facts, and a complete inference over the entire KB would not scale to its size. Although several meaningful paths may exist between two entities, there are a large number of paths which are not meaningful in a specific context. For instance, the reasoning path which goes through path (1) at Fig. 2 is not related or relevant to the classification goal of the entity pairs (the relation between Child of human and Cradle) and should be eliminated by the application of the Distributional Navigation Algorithm (DNA) [20,39], which computes the distributional semantic relatedness between the entities and the intermediate entities in the knowledge base path as a measure of semantic coherence. In this case, the algorithm navigates from $e_{1}$ in the direction of $e_{2}$ in the Knowledge Base using distributional semantic relatedness between the target node $e_{2}$ and the intermediate nodes $e_{n}$ as a heuristic method.

\subsection{Neural Entity/Relation Model (NERM)}

The Distributional Navigational Algorithm provides a pre-filtering of the relations maximizing the semantic relatedness 


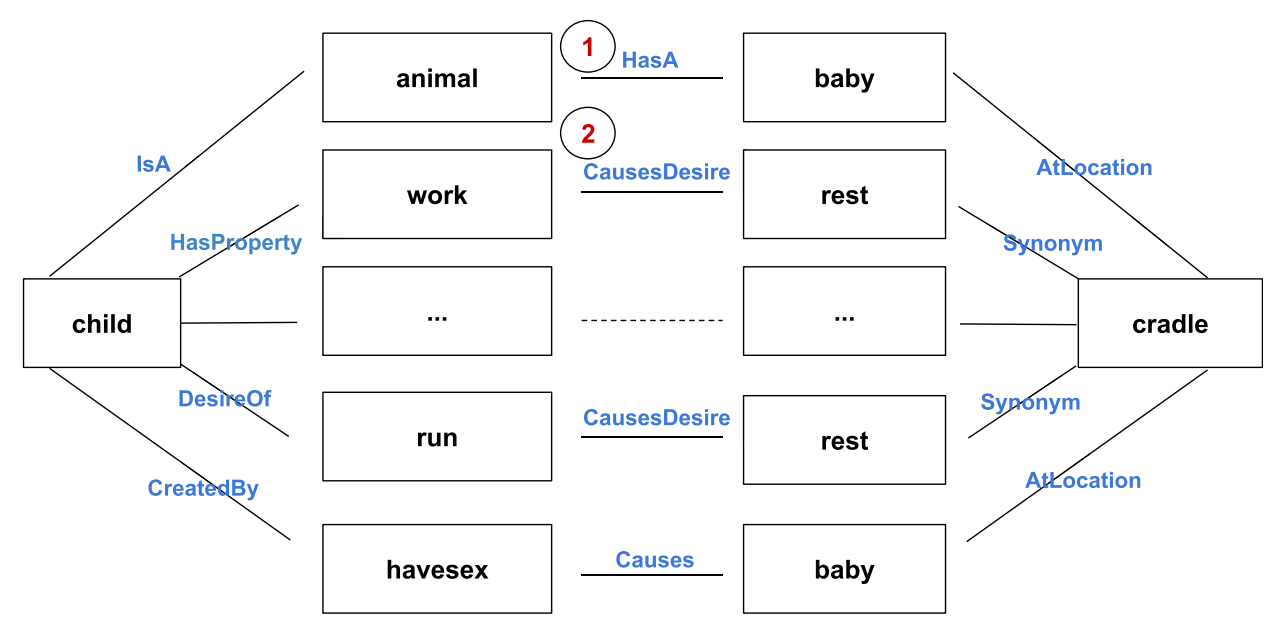

Fig. 2. Selection of semantically relevant paths.

coherence. This can be complemented by a predictive model which takes into account the likelihood of a sequence of relations, i.e. the likelihood of a composition sequence (Algorithm 2). The goal is to systematically compute the sequence of probabilities of entityrelation compositions, in a similar fashion to a language model. As such, the model will capture the notion of a sequence compatibility between entities and relations.

Various machine learning models are used in the context of NLP in order to induce language models and KB-based models, Recursive Neural Networks [42], Recurrent Neural Networks [11,32], Long Short Term Memory Networks [26], Neural Tensor Networks [41] and Convolutional Neural Networks [28]. In this work, due to the nature of the prediction task, which is similar to the language model (identifying semantically coherent sequences of relations), we will target recurrent sequence-classification types of models (see Fig. 4).

\section{Algorithm 2}

Composite Semantic Relation Classification.

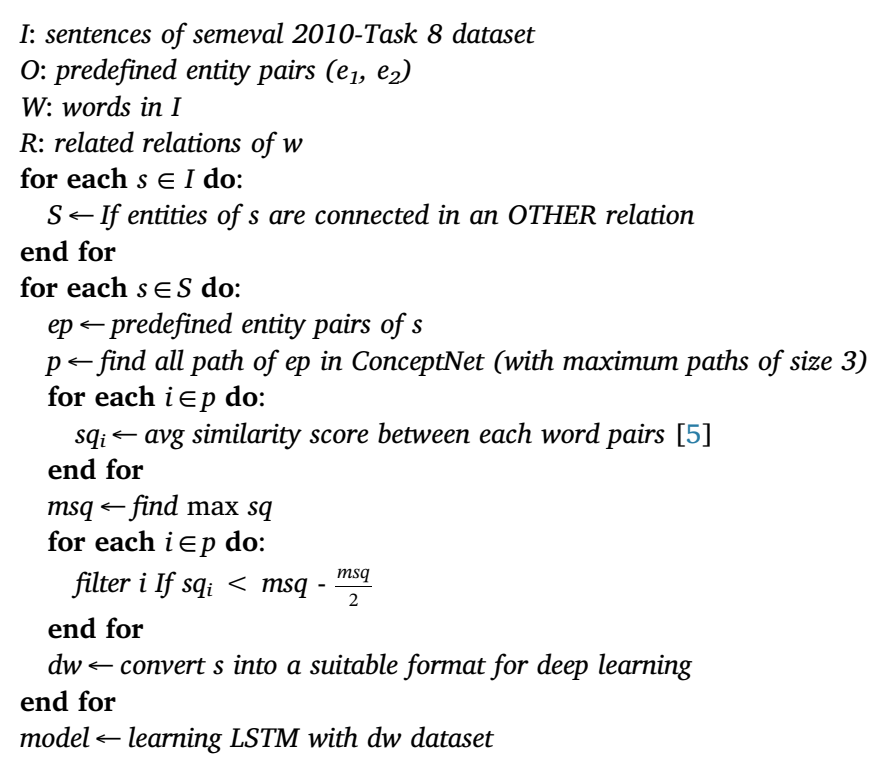

Long Short-Term Memory (LSTM) [26] is a type of recurrent neural network $(R N N)$ architecture whose advantages over other RNN models have been proved in different tasks in NLP $[4,10,45]$. The advantage of LSTM against RNN is that allows the network to capture information from inputs for a long time using a special hidden unit $\left(z_{t}\right)$.

An LSTM unit at a timestep $t$ is described by the following equations:

$$
i_{t}=\delta\left(W_{x, i} x_{t}+W_{z, i} z_{i-1}\right)(\text { input gate })
$$




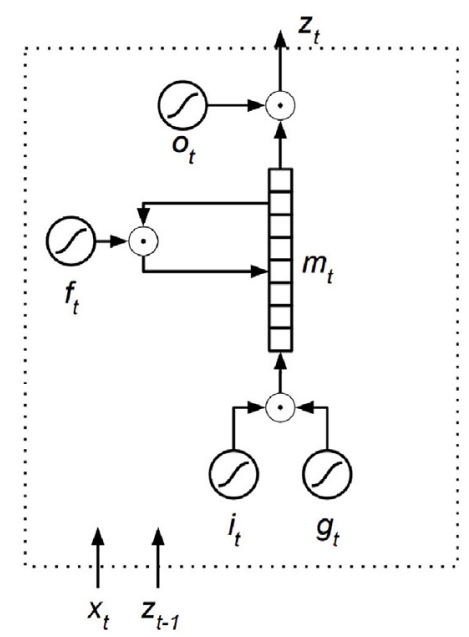

Fig. 3. Long Short-Term Memory unit at timestep $t$. (Small circles with dots are elementwise vector multiplications).

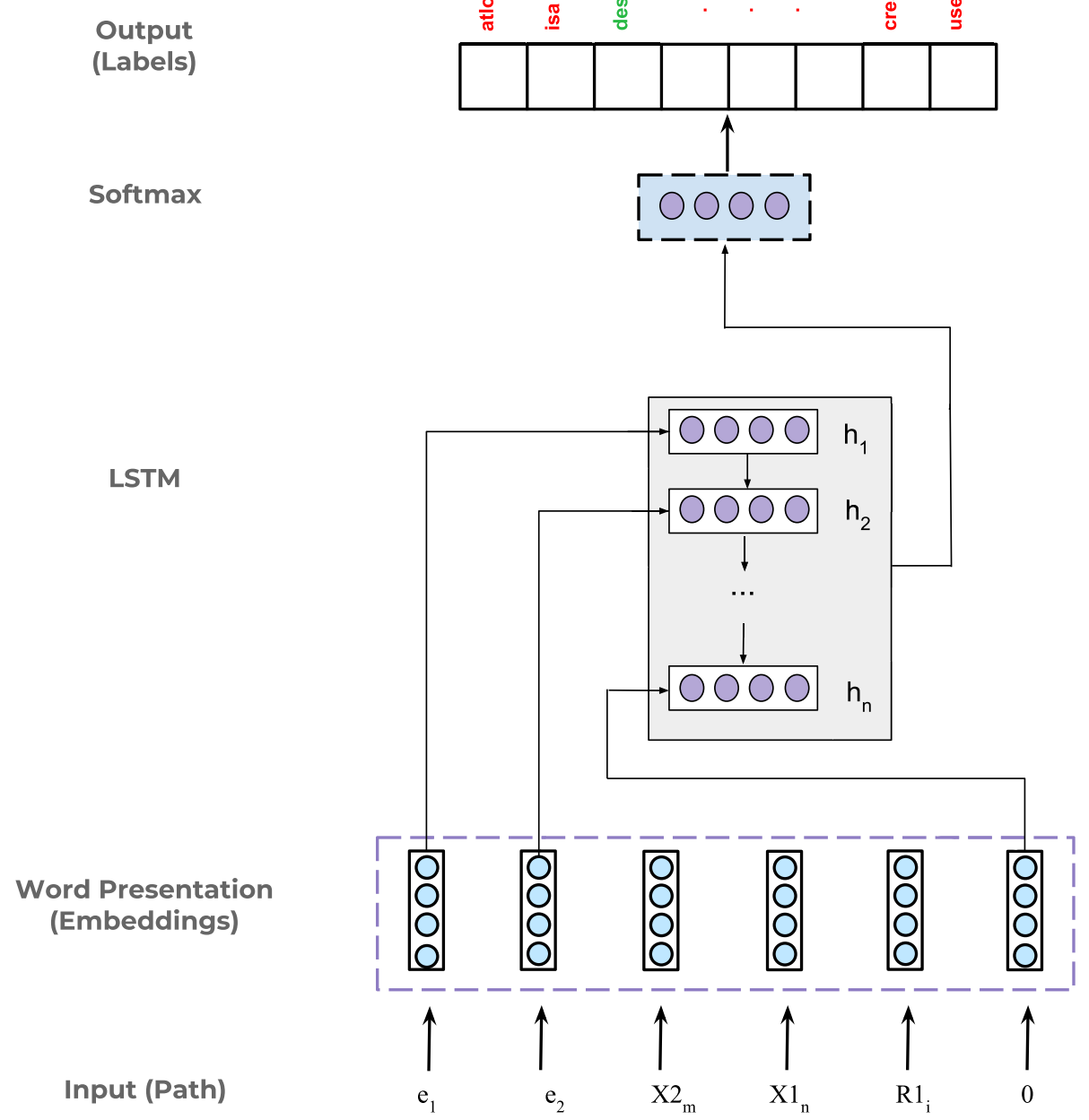

Fig. 4. The Neural Entity/Relation Model Architecture. 


$$
\begin{aligned}
& f_{t}=\delta\left(W_{x, f} x_{t}+W_{z, f} z_{f-1}\right) \text { (forgot gate) } \\
& o_{t}=\delta\left(W_{x, o} x_{t}+W_{z, o} z_{o-1}\right)(\text { output gate }) \\
& g_{t}=\tanh \left(W_{x, g} x_{t}+W_{z, g} z_{g-1}\right)(\text { input modulation gate }) \\
& m_{t}=f_{t} \circ m_{t-1}+i_{t} \circ g_{t}(\text { memory cell) } \\
& z_{t}=o_{t} \circ \tanh \left(m_{t}\right) \text { (hidden state) }
\end{aligned}
$$

The memory vector $m_{t}$ is a function of two parts: (1) its previous value $m_{t-1}$ modulated by the forgot gate $f_{t}(2)$ the information of the current input $x_{t}$ and previous hidden state $\left(z_{t}\right)$ which modulated by the input modulation gate $\left(g_{t}\right)$. Long Short-Term Memory unit at timestep $t$ has four nonlinearity nodes $\left(i_{t}, g_{t}, f_{t}\right.$, and $\left.o_{t}\right)$ all have, as inputs, $x_{t}$ and $z_{t-1}[29,35]$. LSTM can memorize long sequences. The model's input is a sequence of entities and their relations with a specific order. For example, an input for our LSTM model is child - cradle - baby - cause - havesex - creadtedby which model should predict atlocation label.

The model is depicted graphically in Fig. 3.

\section{Experimental evaluation}

\subsection{Training and test dataset}

Two evaluation sets were generated by collecting all pairs of entity mentions in the SemEval 2010 task 8 [25]. The first dataset consists of entity pairs that have no attached semantic relation classes (i.e. which contained the relation label OTHER) while the second dataset contains ALL relations including relations labelled with OTHER. ${ }^{7}$

For all entities, we did a ConceptNet lookup [44], where we generated all paths from lengths 1, 2 and 3 (number of relations) occurring between both entities $\left(e_{1}\right.$ and $\left.e_{2}\right)$ and their relations $(R)$.

For example:

$$
\begin{aligned}
& \mathbf{e} 1-R 1_{i}-\mathbf{e} 2 \\
& \mathbf{e} 1-R 1_{i}-\mathbf{X} 1_{n}-R 2_{j}-\mathbf{e} 2 \\
& \mathbf{e} 1-R 1_{i}-\mathbf{X} 1_{n}-R 2_{j}-\mathbf{X} 2_{m}-R 3_{k}-\mathbf{e} 2
\end{aligned}
$$

where $X$ contains the intermediate entities between the target entity mentions e1 and e2. Obviously, between two entities there may be different paths expressed with different intermediary entities and relations. For instance, for the paths between silver and ring entities we have:

\footnotetext{
- silver/UsedFor/jewelry/MadeOf/gold/AtLocation/ring

- silver/Antonym/gold/AtLocation/ring

- silver/Antonym/bronze/Antonym/gold/AtLocation/ring

- silver/AtLocation/jewelry/MadeOf/gold/AtLocation/ring
}

In next step, the Distributional Navigational Algorithm (DNA) is applied over the entity paths [20,39]. In the final step of generating the training \& test datasets from 3, 728 entity pairs assigned to OTHER relation label in SemEval (OTHER dataset), we found 20, 261 paths and from 21, 434 entity pairs assigned to ALL relations in SemEval (ALL data set), we found 111, 526 paths in ConceptNet.

All paths were converted into the different formats with a specific order of entities and relations, which will be input for Neural Entity/Relation Model (Tables 2-4). ${ }^{8}$ After converting to the new format for our Neural Entity/Relation Model (NERM), we find that we have 25, 260 and 141, 397 unique example relations for both datasets: OTHER and ALL, respectively.

\subsection{Baseline models}

The performance of baselines is measured using the test dataset, as defined in Section 4.1 where we hold out the last relation and rate a system by its ability to infer this relation.

As baselines, we use language models which define the conditional probabilities between a sequence of semantic relations $r$ after the observation of entities $e$, i.e. $P(r \mid e)$.

- Random Model: This is the simplest baseline, which outputs randomly selected relation pairs.

- Unigram Model: Predicts the next relation based on unigram probability of each relation which was calculated from the training set. In this model, relations are assumed to occur independently.

- Bigram Model:

\footnotetext{
${ }^{7}$ Called OTHER and ALL sets, respectively.

${ }^{8}$ The best format based on our experiments is Table 2 .
} 
Table 2

First evaluation dataset for Neural Entity/Relation Model.

\begin{tabular}{lllllll}
\hline \multicolumn{2}{l}{ Input } & & & & & Prediction \\
\hline $\mathbf{e}_{1}$ & $\mathbf{e}_{2}$ & $\mathbf{X} 1_{n}$ & & & & $\mathbf{R} 1_{i}$ \\
$\mathbf{e}_{1}$ & $\mathbf{e}_{2}$ & $\mathbf{X} 2_{m}$ & $\mathbf{X} 1_{n}$ & $\mathbf{R} 1_{i}$ & & $\mathbf{R} 2_{i}$ \\
$\mathbf{e}_{1}$ & $\mathbf{e}_{2}$ & $\mathbf{X} 2_{m}$ & $\mathbf{R 2}_{i}$ & $\mathbf{X} 1_{n}$ & $\mathbf{R} 1_{i}$ & $\mathbf{R} 3_{i}$ \\
\hline
\end{tabular}

Table 3

Second evaluation dataset for Neural Entity/Relation Model.

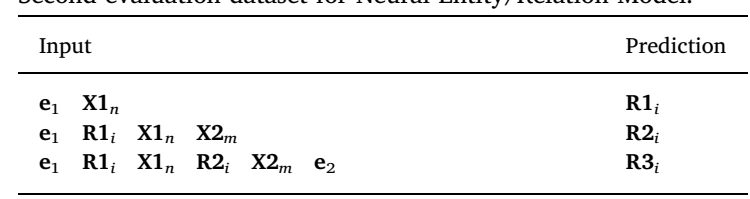

Table 4

Third evaluation dataset for Neural Entity/Relation Model.

\begin{tabular}{lllllll}
\hline \multicolumn{2}{l}{ Input } & & & Prediction \\
\hline $\mathbf{e}_{2}$ & $\mathbf{e}_{1}$ & $\mathbf{X} 1_{n}$ & & & & $\mathbf{R} 1_{i}$ \\
$\mathbf{e}_{2}$ & $\mathbf{e}_{1}$ & $\mathbf{R 1}_{i}$ & $\mathbf{X} 1_{n}$ & & $\mathbf{R 2}_{i}$ \\
$\mathbf{e}_{2}$ & $\mathbf{e}_{1}$ & $\mathbf{R 1}_{i}$ & $\mathbf{X} 1_{n}$ & $\mathbf{R 2}_{i}$ & $\mathbf{X} 2_{m}$ & $\mathbf{R 3}_{i}$ \\
\hline
\end{tabular}

The Bigram model is defined by Ref. [27]:

$$
P(r \mid e)=\frac{P(r, e)}{P(e)}
$$

where $P(r \mid e)$ is the probability of seeing $e$ and $r$, in order. Let $A$ be an ordered list of relations and entities, $|A|$ is the length of R, For $i=1, \ldots,|A|$, define $a_{i}$ to be the $i$ th element of $\mathrm{A}$. We rank candidate relations $\mathrm{r}$ by maximizing $\mathrm{P}(\mathrm{r}$, a), defined as

$$
P(r, a)=\sum_{i=1}^{|A|-1} \log P\left(r \mid a_{i}\right)
$$

where the conditional probabilities $P\left(r \mid a_{i}\right)$ calculated using Equation (1).

- Random Forest: is an ensemble learning method for classification and other tasks, that operates by constructing a multitude of decision trees at training time. Random decision forests correct for the decision trees' limitation of overfitting to their training set.

\subsection{Prediction task}

The Neural Entity/Relation Model predicts composite relations between two given entities ( $\mathbf{e}_{1}$ and $\mathbf{e}_{2}$ ). Given a sequence of source and target entities and a sequence of relations between them, the task consists of the prediction of the next relation $X_{i}$.

A semantically relevant path $\mathbf{e}_{1}-R 1_{i}-\mathbf{X} 1_{n}-R 2_{j}-\mathbf{X} 2_{m}-R 3_{k}-\mathbf{e}_{2}$ is converted into the following formats for the classification task (for different path lengths):

We provide statistics for the generated datasets in Table 5, whereby our dataset is divided into a training set and a test set with scale $(80-20 \%)$. Also we used $20 \%$ of the training set for cross-validation. For the OTHER dataset, we have 16 , 166 examples for training, 4, 042 for validation and 5, 952 for testing and for $A L L$ set we have 90, 493 examples for training, 22, 624 for validation and 28,280 for testing.

\subsection{Word embeddings}

The experimental setup consists of the instantiation of the W2V distributional semantic model. Word2Vector (W2V) [31] provides

Table 5

Distribution of instances used to train the LSTM model.

\begin{tabular}{llll}
\hline Dataset & \#Train & \#Dev & \#Test \\
\hline OTHER & 16,166 & 5,052 & 4,042 \\
ALL & 90,493 & 28,280 & 22,624 \\
\hline
\end{tabular}


an efficient implementation of the continuous bag-of-words and skip-gram models for computing vector representations of words. These representations can be subsequently used in many natural language processing applications and for further research. INDRA $[15,37]$ provides a software infrastructure which facilitates the experimentation and customization of multilingual Word Embedding Models [2,3], allowing end-users and applications to consume and operate over multiple word embedding spaces as a service. In the experimental setup, we used INDRA as a service to get word embeddings for our classification model.

\subsection{Results}

To achieve the classification goal, we generated a Neural Entity/Relation Model for the composite relation classification task. In our experiments, a batch size 25, with 50 epochs was generated. An embedding layer was defined using Word2Vec pre-trained vectors.

In our experiment, we optimized the hyper-parameters of the LSTM model. After several preliminary experiments, the best model was achieved with the following set of parameters:

- Input length and dimension are 6 and 300, respectively.

- Three hidden layers with 450, 200 and 100 nodes and Tanh activation,

- Dropout technique (0.5),

- Adam optimizer.

We configured our Neural Entity/Relation Model and conducted experiments with three different pre-trained embedding settings:

- Word2Vec (Google News) with 300 dimensions

- Word2Vec (Wikipedia 2016) with 30 dimensions

- No pre-trained word embedding

The accuracy for the configuration above after 50 epochs is shown in Table 6. Table 7 contains the Precision, Recall, F1-Score and Accuracy metrics.

Table 7 shows the comparative analysis between NERM and the existing baselines.

Between the evaluated models, the Neural Entity/Relation Model achieved the highest F1 Score and Accuracy. The Bigram model achieved the second highest accuracy 0.3793 followed by Random forest model 0.3299. The LSTM approach provides an improvement of $9.86 \%$ on accuracy over the baselines, and $11.31 \%$ improvement on the F1-score. Random Forest achieved the highest precision, while the Entity/Relation Model achieved the highest recall.

The extracted information from the confusion matrix is shown in Tables 8 and 9. These two tables are calculated based on the first version of our evaluation, in which we have 3, 120 examples for training, 551 for validation and 1, 124 for testing. In Table 8 the 'Correctly Predicted' column indicates the proportion of relations that are predicted correctly, and 'Correct Prediction Rate' column indicates the rate at which the relations correctly predicted. For instance, our model predicts the relation 'NotIsA' correctly in $100 \%$ of the cases.

Table 9 shows the relations which are wrongly predicted ('Wrongly Predicted' columns). Based on the results, the most incorrectly predicted relation is 'IsA', which accounts for a large proportion of relations of the dataset (around 150 out of 550). In second place is the 'AtLocation' relation (172 out of 550). In third place is the 'antonym' relation. On the other hand, some relations which are not correctly predicted can be treated as semantically equivalent to their prediction, whereby their correct assignment depends on modeling decisions in the relation schema. The same situation occurs for specialization relations (e.g. 'EtymologicallyDerivedFrom' and 'DerivedFrom'). Another issue is the low occurrence of certain relations expressed in the dataset.

\subsection{Enriching relationships}

Based on the results at Table 9, some relations which are not correctly predicted can be treated as semantically equivalent to their prediction. Table 10 contains a description of a set of merged relations (merging more specific relations into more abstract categories with similar semantic function).

Also to keep the datasets coherent, we eliminate vague relations, such as ('RelatedTo'. 'DistinctFrom', 'EtymologicallyRelatedTo') and relations implying negation, such as ('Antonym' and 'Not'). Table 11 shows the accuracy of CSRC(Composite Semantic Relation Classification) after merging relations that are semantically equivalent. Before merging similar relations, OTHER and ALL datasets contained 41 and 44 relations while after merging we have 18 and 21 relations, respectively.

Table 6

Validation accuracy.

\begin{tabular}{lll}
\hline CRSC & W2V Google News & W2V Wikipedia \\
\hline Accuracy & 0.4208 & 0.3841 \\
\hline
\end{tabular}


Table 7

Evaluation results on baseline models and our approach, with four metrics.

\begin{tabular}{|c|c|c|c|c|}
\hline Method & Recall & Precision & F1-Score & Accuracy \\
\hline Random & 0.0160 & 0.0220 & 0.0144 & 0.0234 \\
\hline Unigram & 0.0270 & 0.0043 & 0.0074 & 0.1606 \\
\hline Bigram & 0.2613 & 0.2944 & 0.2502 & 0.3793 \\
\hline Random Forest & 0.2476 & 0.3663 & 0.2766 & 0.3299 \\
\hline Entity/Relation model & 0.3073 & 0.3281 & 0.3119 & 0.4208 \\
\hline
\end{tabular}

Table 8

Extracted information from the Confusion Matrix - Part 1.

\begin{tabular}{|c|c|c|}
\hline Relation & \#Correct Predicted & Correct Predicted Rate \\
\hline NotIsA & 2 & 1 \\
\hline AtLocation & 172 & 0.67 \\
\hline NotDesires & 6 & 0.666 \\
\hline Similar & 5 & 0.625 \\
\hline Desires & 36 & 0.593 \\
\hline HasPrerequest & 23 & 0.547 \\
\hline CausesDesire & 17 & 0.548 \\
\hline IsA & 147 & 0.492 \\
\hline Antonym & 68 & 0.492 \\
\hline InstanceOf & 46 & 0.479 \\
\hline UsedFor & 47 & 0.475 \\
\hline DesireOf & 5 & 0.5 \\
\hline HasContext & 2 & 0.5 \\
\hline HasLastSubevent & 2 & 0.5 \\
\hline NotHasA & 1 & 0.5 \\
\hline MemberOf & 1 & 0.5 \\
\hline HasA & 24 & 0.393 \\
\hline HasSubEvent & 12 & 0.378 \\
\hline PartOf & 16 & 0.374 \\
\hline HasPropertry & 12 & 0.375 \\
\hline Synonym & 54 & 0.312 \\
\hline DerivedFrom & 20 & 0.307 \\
\hline EtymologicallyDerivedFrom & 6 & 0.3 \\
\hline CapableOf & 13 & 0.26 \\
\hline MotivationByGoal & 3 & 0.25 \\
\hline ReceiveAction & 5 & 0.238 \\
\hline CreatedBy & 4 & 0.2 \\
\hline MadeOf & 3 & 0.16 \\
\hline Causes & 3 & 0.15 \\
\hline Genre & 1 & 0.11 \\
\hline
\end{tabular}

\subsection{Knowledge Base (KB) embeddings}

The last part of the evaluation concentrates on assessing the impact of Knowledge Base (KB) embeddings into the ERM model.

\subsubsection{Post-Processing Word Embeddings}

Faruqui et al. [12] proposed a graph-based learning technique to obtain higher quality word embeddings by using lexical relational resources such as Wordnet [13], Freebase [6]. This technique known as retrofitting, brings semantically similar words close together while keeping them (relatively) close to their initial distributional vectors. It is a post-processing approach, whereby we inject semantic constraints into existing distributional vector spaces.

Speer et al. [43] introduced an ensemble method known as ConceptNet-Numberbatch, which combines data from pre-trained word embeddings and knowledge graphs, using a variation on retrofitting [12] to produce a high-quality word embeddings. They achieve this goal by applying the following method:

- Expanding the retrofitting algorithm [12] to benefit from structured links outside the original vocabulary.

- Using ConceptNet [44] as a resource of structured connections between words.

- Merging two pre-trained DSMs (Word2Vec and Glove) using a local linear interpolation. This combination performs better than each of the models separately.

- Applying expanded retrofitting method on the combined vector space model by using ConceptNet as a lexical relational resource.

Speer et al. called their word embedding ConceptNet-Numberbatch, showing that the combined embedding outperforms $W 2 \mathrm{~V}$ on 
Table 9

Extracted information from the Confusion Matrix - Part 2.

\begin{tabular}{|c|c|c|c|c|c|c|c|c|}
\hline Relation & $\begin{array}{l}\text { \# Correct } \\
\text { Predicted }\end{array}$ & Rate & Wrong Relation 1 & $\begin{array}{l}\text { \# Falsely } \\
\text { Predicted for } \\
\text { Relation } 1\end{array}$ & Wrong Relation 2 & $\begin{array}{l}\text { \# Falsely } \\
\text { Predicted for } \\
\text { Relation } 2\end{array}$ & Wrong Relation 3 & $\begin{array}{l}\text { \# Falsely } \\
\text { Predicted for } \\
\text { Relation } 3\end{array}$ \\
\hline AtLocation & 172 & 0.67 & Antonym & 20 & UsedFor & 17 & & \\
\hline Desire & 36 & 0.593 & IsA & 6 & CapableOf & 6 & UsedFor & 5 \\
\hline HasPrerequest & 23 & 0.547 & Synonym & 4 & Antonym & 3 & AtLocation & 2 \\
\hline CausesDesire & 17 & 0.548 & UsedFor & 7 & & & & \\
\hline IsA & 147 & 0.492 & AtLocation & 26 & Antonym & 22 & InstanceOf & 22 \\
\hline Antonym & 68 & 0.492 & IsA & 17 & AtLocation & 9 & & \\
\hline InstanceOf & 46 & 0.479 & IsA & 27 & AtLocation & 8 & & \\
\hline UsedFor & 47 & 0.475 & AtLocation & 26 & IsA & 18 & & \\
\hline HasA & 24 & 0.393 & Antonym & 11 & UsedFor & 6 & & \\
\hline HasSubEvent & 12 & 0.378 & Causes & 5 & Antonym & 4 & & \\
\hline PartOf & 16 & 0.374 & Synonym & 12 & Antonym & 3 & HasProperty & 3 \\
\hline HasProperty & 12 & 0.375 & IsA & 8 & & & & \\
\hline Synonym & 54 & 0.312 & IsA & 31 & HasProperty & 17 & AtLocation & 12 \\
\hline DerivedFrom & 20 & 0.307 & IsA & 10 & Synonym & 8 & $\begin{array}{l}\text { Etymologically- } \\
\text { DerivedFrom }\end{array}$ & 8 \\
\hline $\begin{array}{l}\text { Etymologically- } \\
\text { DerivedFrom }\end{array}$ & 6 & 0.3 & DerivedFrom & 6 & & & & \\
\hline CapableOf & 13 & 0.26 & UsedFor & 13 & IsA & 7 & & \\
\hline MotivatedByGoal & 3 & 0.25 & Causes & 3 & HasSubEvent & 2 & & \\
\hline ReceiveAction & 5 & 0.238 & AtLocation & 9 & UsedFor & 3 & & \\
\hline CreatedBy & 4 & 0.2 & Antonym & 6 & IsA & 5 & & \\
\hline MadeOf & 3 & 0.16 & IsA & 7 & Antonym & 3 & HasA & 2 \\
\hline Causes & 3 & 0.15 & CausesDesire & 6 & HasSubEvent & 4 & DerivedFrom & 3 \\
\hline
\end{tabular}

Table 10

Merging similar relations with a more abstract relation.

\begin{tabular}{ll}
\hline Main Relation & Similar Relations \\
\hline HasSubevent & HasFirstSubevent, HasLastSubevent, HasPrerequisite, Entails, MannerOf \\
Causes & MotivatedByGoal, CausesDesire \\
DerivedFrom & FormOf \\
SimilarTo & Synonym \\
IsA & InstanceOf, DefinedAs \\
LocatedNear & AtLocation, HasA, MadeOf, PartOf \\
\hline
\end{tabular}

Table 11

Accuracy before and after merging similar relations.

\begin{tabular}{llll}
\hline Word Embedding & Without Merging Relations OTHER Set & Merged Similar Relations OTHER Set & Merged Similar Relations ALL Set \\
\hline W2V Google News & 0.42 & 0.64 & 0.73 \\
\hline
\end{tabular}

word-similarity evaluations.

In this work, we used ConceptNet-Numberbatch DSM as a pre-trained embedding variation instead of W2V model. Table 12 shows the accuracy of the CSRC classification using the ConceptNet-Numberbatch word embedding.

Speer et al. consider the data in ConceptNet as a symmetric matrix of association between words to apply the expanded retrofitting method. Therefore, they eliminate non-symmetric relations in ConceptNet and disregard these relation types to generate new word embeddings. We argue that in order to achieve a high quality semantic relation classification, all relations must be taken into account. Hence a more comprehensive approach is needed which includes knowledge about how both asymmetric and symmetric allowing us to inject all semantic constraints into existing word embeddings for completeness.

Table 12

Applying ConceptNet-Numberbatch as a pre-trained embedding vector space model in the CSRC classification model.

\begin{tabular}{llc}
\hline Word Embedding & Merged Similar Relations OTHER Set & Merged Similar Relations ALL Set \\
\hline ConceptNet Numberbatch & 0.66 & 0.74 \\
\hline
\end{tabular}


Table 13

Use of CTransNet as a pre-trained embedding vector space model in the CSRC classification model.

\begin{tabular}{lll}
\hline Word Embedding & Merged Similar Relations OTHER Set & Merged Similar Relations ALL Set \\
\hline CTransNet & 0.73 & 0.80 \\
\hline
\end{tabular}

Table 14

Comparison of accuracy scores of three types of Word Embeddings in our classification model (NERM).

\begin{tabular}{llll}
\hline Word Embedding & Without Merging Relations OTHER Set & Merged Similar Relations OTHER Set & Merged Similar Relations ALL Set \\
\hline W2V - Google News & 0.42 & 0.64 & 0.73 \\
ConceptNet Numberbatch & N/A & 0.66 & 0.74 \\
CTransNet & N/A & 0.73 & 0.80 \\
\hline
\end{tabular}

\subsubsection{Embedding entities and relations}

As a second KB embedding model, we experimented with translation embedding methods as a pre-trained word embedding method.

Bordes et al. [8] proposed an energy-based model for learning low-dimensional embeddings of entities which is materialized into the TransE model. Relationships are represented as translations in the embedding space. In other words, the basic idea behind the model is, in a triple set $(h, l, t)$ that composes two entities $h, t \in E$ the set of entities and a relationship $l \in L$ (the set of relationships), the embedding of the entity $t$ should be close to the embedding of the head entity $h$ plus some vector that depends on the relationship $l$.

$$
h+l \simeq t
$$

To learn such embeddings, they minimize a margin-based ranking criterion over the training set [49], where the scoring function of TransE is

$$
-\left(2 g_{r}^{a}\left(y_{e 1}, y_{e 2}\right)-2 g_{r}^{b}\left(y_{e 1}, y_{e 2}\right)+\left\|V_{r}\right\|_{2}^{2}\right)
$$

where:

$$
g_{r}^{a}\left(y_{e 1}, y_{e 2}\right)=A_{r}^{T}\left(\begin{array}{l}
y_{e 1} \\
y_{e 2}
\end{array}\right) \quad \text { and } \quad g_{r}^{b}\left(y_{e 1}, y_{e 2}\right)=y_{e 1}^{T} B_{r} y_{e 2}
$$

and $A_{r}^{T}, B_{r}$ are relation-specific parameters and equal to $\left(V_{r}^{T} \quad-V_{r}^{T}\right)$ and $I$, respectively.

The main motivation of the translation-based parameterization is the structure of the hierarchical relationships that are very common in KBs; therefore translations are the best and natural transformations for representing them. Their model relies on a reduced set of parameters as it learns only one low-dimensional vector for each entity and each relationship. The optimization is carried out by stochastic gradient descent (using minibatches), and also the embedding vectors of the entities are normalized. TransE has fewer parameters compare with other approaches, leading to a simplification of the training process and preventing under-fitting.

A new word embedding called CTransNet was build by applying STransE [33] on the ConceptNet semantic network [44]. We trained STransE with ConceptNet-Numberbatch pre-trained word vectors, size $=300,11$ norm, margin $=5$ and learning rate $=0.0005$, nepoch $=2000$ using ConceptNet V 5.5. Table 13 shows the accuracy of the CSRC classification using the CTransNet word embedding.

\subsection{Final results}

In the previous sections, we investigated the influence of different modalities of pre-trained models for the task of composite semantic relation classification in the context of the Neural Entity-Relation Model (NERM). Three models were analyzed: (1) traditional Word vector embeddings (W2V), (2) Post-Processing Word Embeddings and (3) Embedding Models of Entities and Relations.

The results (Table 14) shows that using CTransNet Word embedding outperforms the W2V - Google News and ConceptNet-Numberbatch word embedding on composite semantic relation classification task.

\section{Conclusion}

In this paper. we introduced the task of composite semantic relation classification. The paper proposes a composite semantic relation classification model which combines commonsense KB lookup, a distributional semantics based filter and the application of a sequence-based machine learning model to address the task.

The highest accuracy for the task of composite semantic relation classification was achieved by using Long-Short Term Memory as the sequence-based models and translation-based embeddings as the (Neural Entity-Relation Model). The proposed approach achieved 0.80 accuracy for the task at hand.

ConceptNet is built from nodes representing words or short phrases of natural language and abstract relationships between them. Future work will focus on enriching the relations with syntactic information. One example is the Syntactic Ngrams dataset, which 
contains dependency tree fragments extracted from of the Google Books corpus [22]. It contains a diverse set of relations, with maximal significance on relations between words. The dataset corpus is based on 3.5 million English books (Over 10 billion distinct items). A Syntactic Ngram is a rooted connected dependency tree over $n$ words. For each $n$ words in a sentence, a POS-tag ${ }^{9}$ and basic dependency label for a given headword are provided. With this information, we can collect all SPO ${ }^{10}$ relationships for each given word pairs for training our predict model. Also, we have a plan to compare our proposed LSTM model with other models such as CNN. Finally, additional baseline models such as $\mathrm{SVM}^{11}$ will be also added in.

\section{Appendix A. Supplementary data}

Supplementary data related to this article can be found at https://doi.org/10.1016/j.datak.2018.06.005.

\section{References}

[1] S. Auer, C. Bizer, G. Kobilarov, J. Lehmann, R. Cyganiak, Z. Ives, Dbpedia: a Nucleus for a Web of Open Data, The semantic web (2007), pp. 722-735.

[2] S. Barzegar, B. Davis, M.Z.S. Handschuh, A. Freitas. Semr-11: a Multi-lingual Gold-standard for Semantic Similarity and Relatedness for Eleven Languages.

[3] S. Barzegar, B. Davis, S. Handschuh, A. Freitas, Multilingual semantic relatedness using lightweight machine translation, Semantic Computing (ICSC), 2018 IEEE 12th International Conference on, IEEE, 2018, pp. 108-114.

[4] S. Barzegar, A. Freitas, S. Handschuh, B. Davis, Composite semantic relation classification, International Conference on Applications of Natural Language to Information Systems, Springer, 2017, pp. 406-417.

[5] S. Barzegar, J.E. Sales, A. Freitas, S. Handschuh, B. Davis, Dinfra: a one stop shop for computing multilingual semantic relatedness, Proceedings of the 38th International ACM SIGIR Conference on Research and Development in Information Retrieval, ACM, 2015, pp. 1027-1028.

[6] K. Bollacker, C. Evans, P. Paritosh, T. Sturge, J. Taylor, Freebase: a collaboratively created graph database for structuring human knowledge, Proceedings of the 2008 ACM SIGMOD International Conference on Management of Data, AcM, 2008, pp. 1247-1250.

[7] F. Bond, R. Foster, Linking and extending an open multilingual wordnet, ACL, 2013, pp. 1352-1362 (1).

[8] A. Bordes, N. Usunier, A. Garcia-Duran, J. Weston, O. Yakhnenko, Translating embeddings for modeling multi-relational data, Advances in Neural Information Processing Systems, 2013, pp. 2787-2795.

[9] J. Chen, D. Ji, C.L. Tan, Z. Niu, Unsupervised feature selection for relation extraction, Proceedings of IJCNLP, 2005.

[10] K. Cortis, A. Freitas, T. Daudert, M. Huerlimann, M. Zarrouk, S. Handschuh, B. Davis, Semeval-2017 task 5: fine-grained sentiment analysis on financial microblogs and news, Proceedings of the 11th International Workshop on Semantic Evaluation (SemEval-2017), 2017, pp. 519-535.

[11] J.L. Elman, Finding structure in time, Cognit. Sci. 14 (2) (1990) 179-211.

[12] M. Faruqui, J. Dodge, S.K. Jauhar, C. Dyer, E. Hovy, N.A. Smith, Retrofitting Word Vectors to Semantic Lexicons, arXiv preprint arXiv:1411.4166 (2014).

[13] C. Fellbaum, Wordnet and Wordnets, (2005).

[14] A. Freitas, Schema-agnositc Queries over Large-schema Databases: a Distributional Semantics Approach (Ph.D. thesis), (2015).

[15] A. Freitas, S. Barzegar, J.E. Sales, S. Handschuh, B. Davis, Semantic relatedness for all (languages): a comparative analysis of multilingual semantic relatedness using machine translation, Knowledge Engineering and Knowledge Management: 20th International Conference, EKAW 2016, Bologna, Italy, November 19-23, 2016, Proceedings, vol. 20, Springer, 2016, pp. 212-222.

[16] A. Freitas, E. Curry, S. Handschuh, Towards a distributional semantic web stack, URSW, Citeseer, 2014, pp. 49-52.

[17] A. Freitas, J.C. Da Silva, S. O'Riain, E. Curry, Distributional relational networks, AAAI Fall Symposium Series, 2013.

[18] A. Freitas, S. Handschuh, E. Curry, Distributional-relational models: scalable semantics for databases, 2015 AAAI Spring Symposium Series, 2015.

[19] A. Freitas, J.C.P. da Silva, Semantics at Scale: when Distributional Semantics Meets Logic Programming, ALP Newsletter, 2014.

[20] A. Freitas, J.C.P. da Silva, E. Curry, P. Buitelaar, A distributional semantics approach for selective reasoning on commonsense graph knowledge bases, Natural Language Processing and Information Systems, Springer, 2014, pp. 21-32.

[21] A. Garcia-Duran, A. Bordes, N. Usunier, Y. Grandvalet, Combining two and three-way embedding models for link prediction in knowledge bases, J. Artif. Intell. Res. 55 (2016) 715-742.

[22] Y. Goldberg, J. Orwant, A dataset of syntactic-ngrams over time from a very large corpus of English books, * SEM@ NAACL-HLT, 2013 , pp. 241-247.

[23] Z.S. Harris, Distributional structure, Word 10 (2-3) (1954) 146-162.

[24] T. Hasegawa, S. Sekine, R. Grishman, Discovering relations among named entities from large corpora, Proceedings of the 42nd Annual Meeting on Association for Computational Linguistics, Association for Computational Linguistics, 2004, p. 415.

[25] I. Hendrickx, S.N. Kim, Z. Kozareva, P. Nakov, D. Ó Séaghdha, S. Padó, M. Pennacchiotti, L. Romano, S. Szpakowicz, Semeval-2010 task 8: multi-way classification of semantic relations between pairs of nominals, Proceedings of the Workshop on Semantic Evaluations: Recent Achievements and Future Directions, Association for Computational Linguistics, 2009, pp. 94-99.

[26] S. Hochreiter, J. Schmidhuber, Long short-term memory, Neural Comput. 9 (8) (1997) 1735-1780.

[27] B. Jans, S. Bethard, I. Vulić, M.F. Moens, Skip n-grams and ranking functions for predicting script events, Proceedings of the 13th Conference of the European Chapter of the Association for Computational Linguistics, Association for Computational Linguistics, 2012, pp. 336-344.

[28] Y. Kim, Convolutional Neural Networks for Sentence Classification, arXiv preprint arXiv:1408.5882 (2014).

[29] Y.L. Kong, Q. Huang, C. Wang, J. Chen, J. Chen, D. He, Long short-term memory neural networks for online disturbance detection in satellite image time series, Rem. Sens. 10 (3) (2018) 452.

[30] Y. Liu, F. Wei, S. Li, H. Ji, M. Zhou, H. Wang, A Dependency-based Neural Network for Relation Classification, arXiv preprint arXiv:1507.04646 (2015).

[31] T. Mikolov, K. Chen, G. Corrado, J. Dean, Efficient Estimation of Word Representations in Vector Space, arXiv preprint arXiv:1301.3781 (2013).

[32] T. Mikolov, M. Karafiát, L. Burget, J. Černockỳ, S. Khudanpur, Recurrent neural network based language model, Eleventh Annual Conference of the International Speech Communication Association, 2010.

[33] D.Q. Nguyen, K. Sirts, L. Qu, M. Johnson, Stranse: a Novel Embedding Model of Entities and Relationships in Knowledge Bases, arXiv preprint arXiv:1606.08140 (2016).

[34] T.H. Nguyen, R. Grishman, Combining Neural Networks and Log-linear Models to Improve Relation Extraction, arXiv preprint arXiv:1511.05926 (2015).

[35] K. Pichotta, R.J. Mooney, Using Sentence-level Lstm Language Models for Script Inference, arXiv preprint arXiv:1604.02993 (2016).

[36] P. Qin, W. Xu, J. Guo, An empirical convolutional neural network approach for semantic relation classification, Neurocomputing (2016).

[37] J.E. Sales, L. Souza, S. Barzegar, B. Davis, A. Freitas, S. Handschuh, Indra: a word embedding and semantic relatedness server, Proceedings of the Eleventh International Conference on Language Resources and Evaluation, LREC, 2018.

[38] C.N. dos Santos, B. Xiang, B. Zhou, Classifying relations by ranking with convolutional neural networks, Proceedings of the 53rd Annual Meeting of the

\footnotetext{
${ }^{9}$ Penn-Treebank part of speech tag.

10 Subject, Predicate, and Object.

11 Support Vector Machines.
} 
Association for Computational Linguistics and the 7th International Joint Conference on Natural Language Processing, vol. 1, 2015 , pp. 626-634.

[39] V. Silva, S. Handschuh, A. Freitas, Recognizing and Justifying Text Entailment through Distributional Navigation on Definition Graphs, AAAI, 2018.

[40] P. Singh, et al., The public acquisition of commonsense knowledge, Proceedings of AAAI Spring Symposium: Acquiring (And Using) Linguistic (And World) Knowledge for Information Access, 2002.

[41] R. Socher, D. Chen, C.D. Manning, A. Ng, Reasoning with neural tensor networks for knowledge base completion, Advances in Neural Information Processing Systems, 2013, pp. 926-934.

[42] R. Socher, A. Perelygin, J. Wu, J. Chuang, C.D. Manning, A. Ng, C. Potts, Recursive deep models for semantic compositionality over a sentiment treebank, Proceedings of the 2013 Conference on Empirical Methods in Natural Language Processing, 2013, pp. 1631-1642.

[43] R. Speer, J. Chin, C. Havasi, Conceptnet 5.5: an open multilingual graph of general knowledge, AAAI, 2017, pp. 4444-4451.

[44] R. Speer, C. Havasi, Representing general relational knowledge in conceptnet 5, LREC, 2012, pp. 3679-3686.

[45] I. Sutskever, O. Vinyals, Q.V. Le, Sequence to sequence learning with neural networks, Advances in Neural Information Processing Systems, 2014, pp. 3104-3112.

[46] K. Xu, Y. Feng, S. Huang, D. Zhao, Semantic Relation Classification via Convolutional Neural Networks with Simple Negative Sampling, arXiv preprint arXiv:1506.07650 (2015).

[47] Y. Xu, R. Jia, L. Mou, G. Li, Y. Chen, Y. Lu, Z. Jin, Improved Relation Classification by Deep Recurrent Neural Networks with Data Augmentation, arXiv preprint arXiv:1601.03651 (2016)

[48] Y. Xu, L. Mou, G. Li, Y. Chen, H. Peng, Z. Jin, Classifying relations via long short term memory networks along shortest dependency paths, Proceedings of Conference on Empirical Methods in Natural Language Processing (To Appear), 2015.

[49] B. Yang, W.t. Yih, X. He, J. Gao, L. Deng, Embedding Entities and Relations for Learning and Inference in Knowledge Bases, arXiv preprint arXiv:1412.6575 (2014).

[50] D. Zeng, K. Liu, S. Lai, G. Zhou, J. Zhao, et al., Relation classification via convolutional deep neural network, COLING, 2014, pp. 2335-2344.

[51] T. Zesch, C. Müller, I. Gurevych, Extracting lexical semantic knowledge from wikipedia and wiktionary, LREC, vol. 8, 2008 , pp. 1646-1652.

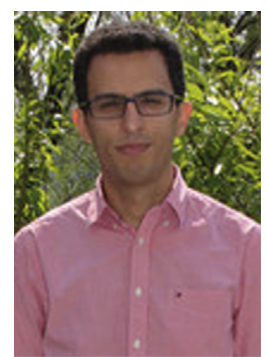

Siamak Barzegar is a final year PhD candidate at Insight Centre for Data Analytics, former Digital Enterprise Research Institute at the National University of Ireland, Galway under the supervision of Dr Andre Freitas and Dr Brian Davis. He won the Science Foundation Ireland (SFI) research scholarship to undertake his $\mathrm{PhD}$ in the area of Scalable Knowledge Extraction, Capturing and Discovery. The main area of his $\mathrm{PhD}$ research is focusing on generating a Distributional Semantics (Word Embedding) Architecture that is transportable across different domains and languages. During his $\mathrm{PhD}$, for near two years, he was part of Prof. Handschuh's research group at the University of Passau (Germany) as a research placement. His research interests include Natural Language Processing, Distributional Semantics, Word Embeddings, Deep Learning, Machine Translation, Knowledge Extraction on Specific Domain. Before joining at the Insight Centre, Siamak worked at Telecommunication Infrastructure Company as a data analyst in fraud detection as a data analyst in fraud detection.

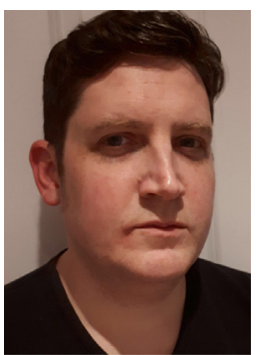

Brian Davis is currently a Lecturer(ATB) in Computer Science at Maynooth University, Co Kildare, Ireland. Prior to taking up this appointment, he was a Research Fellow, Adjunct Lecturer and Research Unit Leader at the INSIGHT Center for Data Analytics, NUIGalway (NUIG), where he led the Knowledge Discovery Unit focusing on the specific research areas of: Natural Language Processing, Data Visualization and Knowledge Discovery from heterogeneous data sources (text and graph). He was coordinator of a 3 year Horizon 2020 Innovation Action - SSIX - Social Sentiment Financial Indexes (Grant No. 645425). His core expertise intersects with Natural Language Processing, Ontology Engineering and Data Visualization. Other research interests include: NLP for social media, cross lingual opinion mining from social media for the finance and political domains and combining visualization and textualisation using Natural Language Generation. He has over eight years research experience in Ontology Based Information Extraction and Semantic Annotation.

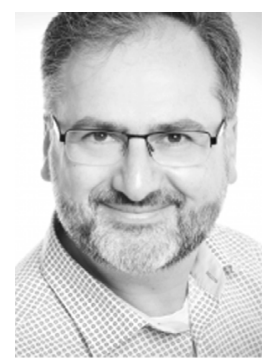

Prof. Dr. Siegfried Handschuh is professor at Universität Passau, Fakultät für Informatik und Mathematik, Lehrstuhl für Informatik mit Schwerpunkt Digital Libraries and Web Information Systems. Prior to the acquisition of the Chair in Passau, he was professor at the National University of Ireland, Galway (NUIG) and head of the Knowledge Discovery Unit at the Insight Center for Data Analytics in Galway.

He studied computer science in Ulm and information science in Konstanz and was assistant at the Institute for Applied Computer Science and Formal Description Methods at the University of Karlsruhe, subsequently. In 2001, he continued with a research stay at Stanford Database Group at Stanford University in the United States. He received his doctorate with magna cum laude in Karlsruhe in 2005 .

Prof. Handschuh coordinated numerous research and development projects on international level. He was involved in attracting extensive project funding: of the EU, the Science Foundation Ireland, Enterprise Ireland and from national sources, amongst others. In addition, he has worked with multinational companies such as HP, SAP, IBM, Motorola, Cisco, Avaya, British Telecom, Telecom Italia, Telefonica, Thales and Elsevier Publishing.

Furthermore, he conducted research at Digital Aristoteles Project that aimed at the semantic content analysis and knowledge modeling of schoolbooks, and was funded by the Microsoft founder and philanthropist, Paul Allen. His current research deals mainly with the areas of semantic technologies, information linguistics, information extraction and web information retrieval. 


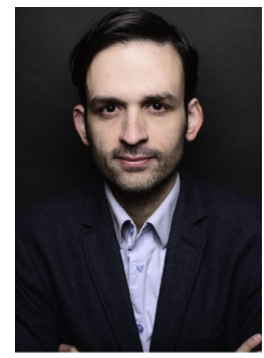

André Freitas is a lecturer (assistant professor) at the School of Computer Science at the University of Manchester. Prior to Manchester, he was an associate researcher and lecturer at the Natural Language Processing and Semantic Computing Group at the University of Passau (Germany) at the Chair of Digital Libraries and Web Information Systems. He is also a partner and co-founder at Amtera Semantic Technologies. Before joining Passau, he was part of the Digital Enterprise Research Institute (DERI) at the National University of Ireland, Galway where he did his PhD on Schema-agnostic Query Mechanisms for Large-Schema Databases. André holds a BSc. in Computer Science from the Federal University of Rio de Janeiro (UFRJ), Brazil (2005). His main research areas include Question Answering, Schema-agnostic Database Query Mechanisms, Natural Language Query Mechanisms over Large-Schema Databases, Distributional Semantics, Hybrid Symbolic-Distributional Models, Approximate Reasoning and Knowledge Graphs. Before joining DERI, André worked as a research assistant (trainee) at Siemens Corporate Research, Princeton, USA. André worked as a software engineer, product designer and project manager in different industries including Oil \& Gas Exploration, IT Security, Medical, Healthcare, Banking, Mining and Telecom. 